

\title{
ENVEJECIMIENTO: CAMBIOS ESTRUCTURALES Y PAPEL DE LA FACILITACIÓN NEUROMUSCULAR PROPIOCEPTIVA
}

\section{Elderly: Structural Changes and the Role of the Neuromuscular Proprioceptive Facilitation}

\author{
Fecha de Recepción: 27 de Marzo de 2012 - Fecha de Aprobación: 16 de Agosto de 2012
}

\author{
RESUMEN
}

En el mundo, el porcentaje de adultos mayores se encuentra en aumento, obligando al desarrollo continuo de políticas en salud. En razón a los cambios morfológicos y estructurales propios del músculo esquelético durante el envejecimiento, que llevan a su deterioro, varios modelos de ejercicio han sido puestos a prueba, incluyendo el entrenamiento de la fuerza explosiva y la flexibilidad. La Facilitación Neuromuscular Propioceptiva, ha surgido como una alternativa de entrenamiento, gracias a los resultados positivos demostrados en el área de la prevención y la rehabilitación; sin embargo en el adulto mayor sano no ha sido posible demostrar dichos beneficios, presentándose por el contrario dificultades en la aplicación de los programas, dados por los tiempos necesarios para hacer tangibles los resultados y los riesgos que con su implementación se puedan presentar. Es necesario desarrollar nuevas investigaciones que involucren técnicas combinadas de entrenamiento, con el fin de comprender y optimizar la respuesta al ejercicio físico en el adulto mayor.

PALABRAS CLAVE

Envejecimiento, Sarcopenia, Flexibilidad, Facilitación Neuromuscular Propioceptiva.

\section{ABSTRAC}

Worldwide the percentage of elderly people is increasing, forcing the continued development of health policies. Due to structural and morphological characteristic changes of skeletal muscle during aging, leading to deterioration, several models of exercising have been tested, including the explosive strength and flexibility training, the Proprioceptive Neuromuscular Facilitation has emerged as an alternative training, because of the good results shown in the prevention and rehabilitation area. However in healthy elderly adults have not been possible to demonstrate those benefits, instead they have revealed difficulties in programs implementation, in part for the time needed to obtain tangible results and the risks involved with its implementation may arise. Furthermore, it is necessary to develop combined techniques involving training, in order to understand and optimize the response to exercise in the elderly.

KEY WORDS

Aging, Sarcopenia, Flexibility, Proprioceptive Neuromuscular Facilitation. 
$\mathrm{E}$ n la actualidad el porcentaje de la población adulta mayor ha aumentado dramáticamente en todo el mundo, hacia 1950 la proporción de adultos mayores era cercana al 8\%, para el año 2009 esta fue del $11 \%$ y se estima que para el año 2050 supere el 22\% (Organización de Naciones Unidas, 2009). En Colombia los resultados de la Encuesta Nacional de Demografía y Salud (ENDS), muestran que la población de adultos mayores de 60 años corresponde al $10 \%$ de la totalidad de habitantes, representando casi 4,5 millones de personas (Profamilia, 2010).

Muchas son las condiciones patológicas que aquejan al adulto mayor; en la actualidad una de las principales causas de incapacidad y morbilidad lo constituyen el riesgo de caídas, ya que más de un tercio de las personas mayores de 65 años ha sufrido al menos una de estas en el último año (Tinetti \& Kumar, 2010), el origen de dichas caídas se asocia fuertemente a la pérdida del control musculoesquelético por parte del sistema nervioso central, alteraciones en la información aferente sensitiva y una disminución en la efectividad del sistema ejecutor muscular (Al-Aama, 2011).

Condiciones como la alta prevalencia de caídas, la disminución en el control neuromuscular, la alteraciones en el balance postural entre otros, obliga a los programas de promoción y prevención a desarrollar estrategias con las que se puedan asegurar en la población adulta mayor; un menor riesgo de lesiones y una adecuada calidad de vida(Clemson et al., 2012), en este sentido, ampliamente se reconoce que una de las intervenciones más benéficas es el ejercicio físico (Nocon et al., 2008), y dentro de esté, especialmente la realización de ejercicios de resistencia y aquellos que llevan a mejorar el balance y la funcionalidad (Weening-Dijksterhuis, de Greef, Scherder, Slaets, \& van der Schans, 2011).

Esta revisión busca presentar inicialmente los cambios que a nivel muscular y de tejidos blandos se presentan en el adulto mayor, y cómo algunas intervenciones terapéuticas asociadas al ejercicio participan en el mejoramiento de la fuerza y la flexibilidad; posteriormente se analizará y se evalúa el papel de la Facilitación Neuromuscular Propioceptiva (FNP) en diferentes ámbitos y poblaciones, y por último a partir de la evidencia se expondrá el papel que juega la FNP en el manejo del adulto mayor. Para todo lo anterior la revisión se basa en la pregunta de investigación: ¿Existe evidencia científica que favorezca la implementación de programas de FNP en la salud integral del adulto mayor?

\section{METODOLOGÍA}

La revisión fue desarrollada utilizando las bases datos facilitadas por la Universidad Nacional de Colombia, la búsqueda fue realizada utilizando las palabras claves: Proprioceptive Neuromuscular Facilitation (PNF), Stretching, Older people y ageing, en un periodo de tiempo que abarcó los últimos 10 años (Enero 2002 - Enero 2012), se incluyeron en la búsqueda artículos originales y revisiones. Se hizo especial énfasis en los artículos que analizaran la influencia de la Facilitación Neuromuscular Propioceptiva en el adulto mayor. El flujograma de búsqueda y análisis de la información se encuentra en la figura 1.

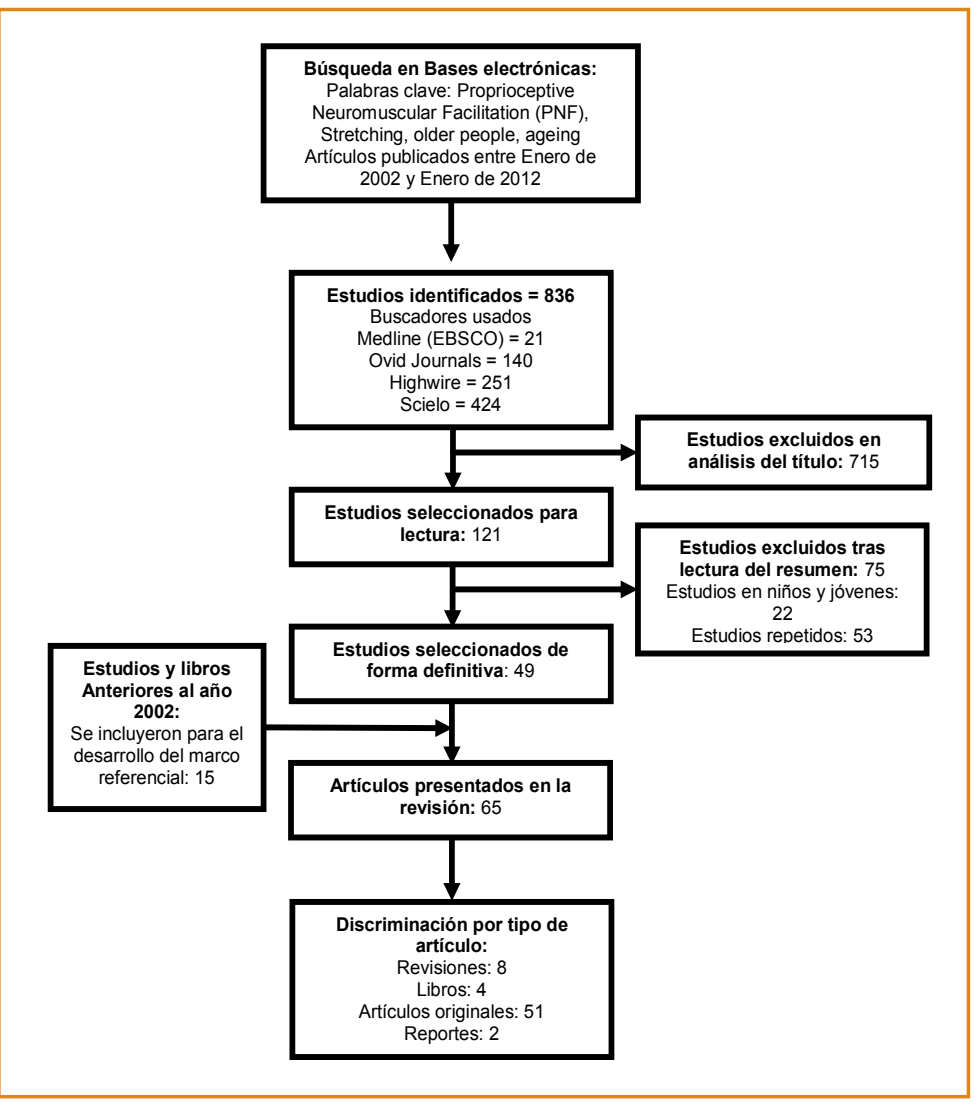

Figura 1. Flujograma de Búsqueda, análisis y utilización de las fuentes bibliográficas consultadas

Como complemento a la búsqueda se incluyeron 4 libros, 2 reportes y 11 artículos anteriores al año 2002, que se consideraron fundamentales por su aporte; al final el número de referencias incluidas fue de 65 .

\section{RESULTADOS}

\section{Cambios Musculares e Intervenciones}

Asociadas al Proceso de Envejecimiento

Es ampliamente aceptado que el proceso natural de envejecimiento en los seres humanos se acompaña de una pérdida progresiva de la función neuromuscular y del rendimiento físico (Vandervoort, 2002), esta disminución de la masa y la calidad del músculo esquelético es un fenómeno habitualmente conocido como sarcopenia(Zhong, Chen, \& Thompson, V, 2007; Doherty, 2003); sin embargo, en la actualidad el término sarcopenia no solo se refiere al músculo, sino también a otras condiciones que afectan su fisiología, como su inervación, el estatus hormonal, los efectos inflamatorios y las alteraciones en la ingesta proteo-calórica (Doherty, 2003). El resultado de la sarcopenia lleva entonces no solo a la disminución de la masa muscular sino también a alteraciones en la producción de fuerza muscular (Doherty, 2003; Frontera, Hugues, Lutz, \& Evans, 1991) y a cambios en las propiedades elásticas del músculo asociadas a una fuerte disminución en el rango de amplitud articular (Gajdosik et al., 2005). 
A la par con cambios en la estructura muscular, el envejecimiento también supone cambios en todo el sistema de movilidad y desplazamiento (sistema osteo-muscular), estos pueden resumirse en los cambios que comprometen las características y propiedades visco-elásticas del tejido conectivo, las cuales se alteran con la edad (Gajdosik et al., 2004) o la disminución de la densidad mineral ósea, la cual también decae (Ettinger, 2003). Ya en varias ocasiones se ha logrado determinar que estos cambios a nivel osteo-muscular se acompañan de alteraciones en la calidad de vida, en una pérdida de la funcionalidad y en un incremento en la posibilidad de caídas y lesiones del adulto mayor (Rebelatto, Castro, \& Paiva, 2007; Lord, Sherrington, Menz, \& Close, 200o; Rubenstein, 2006).

Como un mecanismo eficaz para contrarrestar la sarcopenia y las limitaciones asociadas al envejecimiento, se han venido desarrollando múltiples programas de intervención en torno de la importancia que tiene el desarrollo de la fuerza explosiva en la funcionalidad del adulto mayor (Ramírez, Da Silva, \& Lancho, 2007; Ramírez, 2011); como resultado de dichas investigaciones ha sido posible demostrar que a través del ejercicio físico no sólo es posible en el adulto mayor mejorar la calidad de la masa muscular, aunque sin cambios significativos en hipertrofia (Sipila \& Suominen, 1995), sino que además el entrenamiento de la fuerza puede llevar a una marcada disminución del riesgo cardiovascular (Goodpaster et al., 2008).

Otra área de intervención donde existe un continuo avance de la información está en los programas de ejercicio que se centran en el desarrollo y mejora de la flexibilidad muscular, para ello se parte de la fisiología adaptativa al estiramiento a través de la cual, se ha demostrado que el desarrollo de la flexibilidad lleva a cambios en la morfología de fibras musculares, incremento del número de sarcómeros y reducción en la posibilidad de atrofia (Gomes, Cornachione, Salvini, \& Mattiello-Sverzut, 2007), muchos de estos resultados se han explicado gracias a la activación en la síntesis proteica (Goldspink, 1999), además de adaptaciones en el tejido conectivo, estas últimas son al parecer secundarias a la reorganización de las fibras de colágeno (Coutinho, DeLuca, Salvini, \& Vidal, 2006).

En la etapa de envejecimiento, las adaptaciones fisiológicas secundarias al estiramiento parecen explicar los resultados en el desarrollo de la fuerza encontrados en estudios con adultos mayores, que fueron sometidos a sesiones controladas de estiramiento (Gajdosik, Vander Linden, McNair, Williams, \& Riggin, 2005), además de otros hallazgos, como la mejora en el gesto de la marcha secundaria a programas de flexibilidad, con los que se ha logrado incrementar tanto la longitud como en la velocidad de la misma (Ringsberg, Gerdhem, Johansson, \& Obrant, 1999).

En resumen, los programas encaminados a la mejora de la fuer$\mathrm{za}$, (especialmente la fuerza explosiva) y los programas que incluyen mejoras en la elasticidad muscular a través del estiramiento, que se han llevado a cabo en adultos mayores, han demostrado una gran cantidad de beneficios en el desarrollo muscular y en la calidad de vida.

\section{Facilitación Neuromuscular Propioceptiva (FNP)}

A principios de la década de los noventa, Charles Scott Sherrington, un neurofisiólogo británico, definió el concepto de facilitación e inhibición neuromuscular (Sherrington, 1908), este principio fisiológico fue utilizado posteriormente para el desarrollo de técnicas de estiramiento a través de la FNP por Herman Kabat en 1940 (Kabat, 1947), llevando a que la técnica de FNP fuera usada especialmente en el proceso de rehabilitación de pacientes con parésias y espasticidad; todavía en la actualidad, el principio sigue siendo utilizado en el manejo del paciente con patología neurológica (Sharman, Cresswell, \& Riek, 2006).

La Facilitación Neuromuscular Propioceptiva es considerada por varios autores como el método más efectivo para mejorar el rango de movilidad articular (Shrier \& Gossal, 2000; Sharman et al., 2006; Rowlands, V, Marginson, \& Lee, 2003; Chalmers, 2004; Alter, 2004), combina varios elementos como lo son: la contracción agonistaantagonista, la duración de las fases de relajación y contracción, las relaciones de tiempo entre cada fase, el número e intensidad de las contracciones, entre otras (Alder, Beckers, \& Buck, 2008). Múltiples estudios han demostrado el efecto de utilizar diferentes intensidades de contracción de acuerdo a las necesidades (Magnusson et al., 1996; Feland \& Marin, 2004; Burke, Culligan, Holt, \& McKinnon, 2000).

Desde el punto de vista fisiológico la FNP se basa en el aumento de la respuesta de los mecanismos neuromusculares por estimulación de los propioceptores, y para tal fin se incluyen ejercicios en espiral, diagonal y línea recta en dirección topográfica de los músculos que se utilizan (Voss, Lonta, \& Meyer, 1985).

Las técnicas de FNP han demostrado ser efectivas en el manejo de varias condiciones patológicas: El dolor crónico de espalda; en donde se ha demostrado una mejora en la movilidad y la resistencia muscular lumbar (Kofotolis \& Kellis, 2006), en el manejo de secuelas de accidente cerebrovascular, donde junto con otras técnicas ha sido posible mejorar el déficit sensitivo (Wolny, Saulicz, Gnat, \& Kokosz, 2010), en la calidad de vida de pacientes con enfermedad de Parkinson, donde ha sido posible generar cambios posturales y de marcha (Mirek et al., 2003), en enfermedades que afectan la motoneurona inferior como la Amiotrofia Local Benigna, donde ha sido posible mejorar la fuerza y la coordinación muscular (Orsini et al., 2010). A nivel ortopédico y de rehabilitación la FNP ha sido efectivo como facilitador en la recuperación del rango de movilidad articular luego de trasplantes de rodilla (Chow \& Nq, 2010), en programas de manejo de pacientes con artrosis de rodilla, programas en los cuales la FNP ha demostrado que es posible mejorar el rango de movimiento y la fuerza muscular (Weng et al., 2009) y en los beneficios no solo a nivel muscular sino de tejidos blandos en patologías de la articulación gleno-humeral (Godges, Mattson-Bell, Thorpe, \& Shah, 2003; Mahomed, Al-Obaidi, \& Al-Zoabi, 2008).

En lo que respecta a la mejora de las capacidades motrices en un proceso de entrenamiento deportivo de alto nivel, la FNP también ha demostrado ser efectiva en la reducción del riesgo de lesiones ante contracciones intensas submaximales (Feland \& Marin, 2004) y como profilaxis ante las alteraciones motoras y sensitivas que se presentan luego de contracciones especialmente de tipo excéntrico (Khamwong, Pirunsan, \& Paungmali, 2011). Por otro lado ha demostrado un efecto benéfico en programas de ejercicio, con miras a mejorar la estabilidad postural (Ryann, Rossi, \& Lopez, 2010), y 
1 en aquellos que buscan no solo aumentar el rango de movilidad articular, sino también la eficiencia mecánica de la contracción tanto en miembros inferiores (Rees, Murphy, Watsford, McLachlan, \& Coutts, 2007), como en miembros superiores (Decicco, V \& Fischer, 2005), sin que se vea afectada la producción de fuerza a largo plazo (Higgs \& Winter, 2009).

De la misma forma que múltiples estudios han resaltado ampliamente los beneficios de la FNP, hay estudios que no han permitido encontrar diferencia entre la utilización de la FNP y otras técnicas de estiramiento: la fuerza explosiva evaluada a través de saltos y especialmente saltos contra caída (Drop Jump) no presenta mejora con la utilización de FNP (Yuktasir \& Kaya, 2009), tampoco deportistas cuyo objetivo fundamental es la velocidad han demostrado eficiencia mecánica (Caplan, Rogers, Parr, \& Hayes, 2009), ni aquellos que expresan fuerza máxima a través del entrenamiento (Franco, Signorelli, Trajano, \& Oliveira, 2008; Marek et al., 2005), donde los resultados favorecen incluso más el estiramiento dinámico, sobre otras técnicas como el estiramiento estático y el FNP (Manoel, Harris-Love, Danoff, V, \& Miller, 2008), también poblaciones jóvenes parecen obtener resultados en la flexibilidad sin importar la técnica (Wallmann, Gillis, \& Martinez, 2008).

Todo lo anterior intenta demostrar que la FNP tiene efectos benéficos y múltiples aplicaciones, en ámbitos específicos como la prevención de lesiones y la rehabilitación de las mismas, pero que en busca de la mejora de las capacidades físicas en pacientes sanos sus beneficios no son superiores a los encontrados a través de otras técnicas de estiramiento.

\section{Estiramiento y FNP en el Adulto Mayor}

La literatura acerca de programas de Facilitación Neuromuscular Propioceptiva en adultos mayores (AM) sanos es escasa, varios artículos publicados describen los beneficios en condiciones patológicas como ya se mencionó anteriormente: Lumbalgia crónica, Accidente cerebro vascular, Parkinson (Kofotolis \& Kellis, 2006; Mirek et al., 2003; Wolny et al., 2010)y en patologías degenerativas como las artrosis de rodilla y hombro (Godges et al., 2003; Weng et al., 2009; Mahomed et al., 2008).

En AM sanos, algunos estudios han demostrado que utilizando FNP es posible mejorar la flexibilidad y la fuerza de diferentes grupos musculares, llevando a una mejora en su calidad de vida. Como ejemplo de ello, el resultado de varios trabajos propone que la mejora de la flexibilidad, especialmente sobre los músculos flexores de cadera, genera un impacto positivo sobre la marcha (Kerrigan, XenopoulosOddsson, Sullivan, Lelas, \& Riley, 2003; Watt et al., 2011), de la misma forma en el AM mejorar la flexibilidad en músculos extensores y especialmente flexores de rodilla, también lleva a cambios benéficos en la marcha y en las actividades básicas cotidianas (Feland, Myrer, \& Merrill, 20o1; Feland, Myrer, Schulthies, Fellingham, \& Measom, 2001), por último se ha propuesto también, la importancia que sobre la funcionalidad del AM tiene el estiramiento de los músculos de las piernas (Gajdosik et al., 2005); sin embargo los resultados anteriormente expuestos no han evidenciado un beneficio superior de técnicas como la FNP sobre otras técnicas de estiramiento.
Otro aspecto importante de la FNP en el AM, es que los programas utilizados, han llevado a cambios estadísticamente significativos, en la mejora del rango de movilidad articular tanto en miembros superiores como miembros inferiores, en aproximadamente 15 semanas (Klein, Stone, Phillips, Gangi, \& Hartman, 2002), tiempos que son muy superiores a los necesitados por personas jóvenes, donde los cambios pueden verse en periodos de tiempo de apenas cuatro semanas (Higgs \& Winter, 2009).

Por otro lado, la activación muscular secundaria al estiramiento lleva sin importar la técnica a incrementos no solo en la flexibilidad sino también de la fuerza muscular en el AM, esto especialmente en músculos flexores, pero también en músculos extensores (Batista, Vilar, de Almeida Ferreira, Rebelatto, \& Salvini, 2009; Gallon et al., 2011); sin embargo, algunos trabajos publicados sugieren ser muy cuidadoso en la aplicación de técnicas de FNP en AM debido a las profundas alteraciones en la elasticidad muscular que en ellos se dan (Ferber, Osterning, \& Gravelle, 2002), lo cual se relaciona a una mayor tendencia de lesiones.

\section{DISCUSIÓN}

El ejercicio físico debe constituir una parte fundamental en todas las etapas de la vida del ser humano, claramente nuestro desarrollo evolutivo así lo indica; de esta forma, el ejercicio en el adulto mayor, debe orientarse a solventar las necesidades propias del envejecimiento, tales como la pérdida de la masa muscular, su consecuente disminución en la fuerza y muy relacionado a estos dos, las alteraciones en su funcionalidad, la independencia y la disminución en el riesgo de condiciones patológicas de origen traumático como las caídas.

Como parte del acompañamiento integral del adulto mayor a través del ejercicio físico, múltiples trabajos han demostrado que al mejorar la potencia muscular y la flexibilidad es posible mejorar su calidad de vida, y por tal razón no está mal en pensar que técnicas ampliamente reconocidas en el desarrollo de la flexibilidad como la Facilitación Neuromuscular propioceptiva (FNP), son una buena alternativa para la mejora de la funcionalidad. Sin embargo, a la luz de la evidencia no se ha logrado demostrar que la FNP genere un mayor impacto en capacidades condicionales como la fuerza, la velocidad o la flexibilidad, que el que se obtiene por medio de otras técnicas de estiramiento, esto pensando obviamente en el adulto mayor.

Los resultados de esta revisión, obligan a pensar, que los cambios a nivel de la arquitectura muscular, de la respuesta neuromuscular o de las respuestas adaptativas del adulto mayor, alteran también su adaptación fisiológica al ejercicio; probablemente la forma como los receptores musculares propioceptivos responden a estímulos, sean diferentes con el paso del tiempo; esto en razón de las resultados tan discrepantes cuando se comparan la FNP entre jóvenes y adultos mayores.

Si bien es claro que mejorar la flexibilidad y la fuerza del adulto mayor es benéfico sobre su calidad de vida, a la luz de la evidencia no se recomienda su implementación como modelo de entrenamiento en los adultos mayores, de una parte por los pobres resultados benéficos que la acompañan y de otra por la alta tendencia a lesiones musculares y osteo-articulares que se puedan presentar. 
Queda sin embargo, abierta la posibilidad para el desarrollo de nuevas investigaciones, que intenten dilucidar los mecanismos adaptativos de los músculos de los adultos mayores a modelos de entrenamiento como el de "contraer - estirar", los resultados de estas investigaciones aportaran nueva evidencia, valiosa para la prescripción del ejercicio físico.
AGRADECIMIENTOS

Al grupo de investigación en ciencias aplicadas al ejercicio físico, el deporte y la salud (GICAEDS), y al laboratorio de ciencias morfofuncionales de la facultad de Cultura Física, Recreación y Deporte de la Universidad Santo Tomás, en Bogotá - Colombia.

\section{Referencia:}

\section{Las referencias a otras obras son una parte muy importante en la literatura científica;}

\section{ya que estas permiten conocer más sobre los autores y}

mantener vivas sus voces dentro del texto.

1. Al-Aama, T. (2011). Falls in the elderly: spectrum and prevention. Can.Fam.Physician, 57 (7), 771-776. doi:57/7/771 [pii]. Retrieved from PM:21753098

2. Alder, S. S., Beckers, D., \& Buck, M. (2008). PNF in Practice ( $3^{\circ}$ ed.). Germany: Springer.

3. Alter, M. J. (2004). The Science of Flexibility ( $3^{\circ}$ ed.). Champaing, IL: Human Kinetics.

4. Batista, L. H., Vilar, A. C., de Almeida Ferreira, J. J., Rebelatto, J. R., \& Salvini, T. F. (2009). Active stretching improves flexibility, joint torque, and functional mobility in older women. Am J Phys Med Rehabil, 88(10), 815-822.

5. Burke, D. G., Culligan, C. J., Holt, L. E., \& McKinnon, N. C. (2000). Equipment designed to simulate proprioceptive neuromuscular facilitation flexibility training. I Strength Cond Res, 14(2), 135-139.

6. Caplan, N., Rogers, R., Parr, M. K., \& Hayes, P. R. (2009). The effect of proprioceptive neuromuscular facilitation and static stretch training on running mechanics. I Strength Cond Res, 23(4), 1175-1180.

7. Chalmers, G. (2004). Re-examination of the possible role of Golgi tendon organ and muscle spindle reflexes in proprioceptive neuromuscular facilitation muscle stretching. Sports Biomech, 3(1), 159-183.

8. Chow, T. P., \& Nq, G. Y. (2010). Active, passive and proprioceptive neuromuscular facilitation stretching are comparable in improving the knee flexion range in people with total knee replacement: a randomized controlled trial. Clin Rehabil, 24(10), 911-918.

9. Clemson, L., Fiatarone Singh, M. A., Bundy, A., Cumming, R. G., Manollaras, K., O'Loughlin, P. et al. (2012). Integration of balance and strength training into daily life activity to reduce rate of falls in older people (the LiFE study): randomised parallel trial. BMJ, 345, e4547. Retrieved from PM:22872695

10. Coutinho, W. L., DeLuca, C., Salvini, T. F., \& Vidal, B. C. (2006). Bouts of passive stretching after immobilization of the rat soleus muscle increase collagen macromolecular organization and muscle fiber area. Connect Tissue Res, 47(5), 278-286.
11. Decicco, P., V, \& Fischer, M. M. (2005). The effects of proprioceptive neuromuscular facilitation stretching on shoulder range of motion in overhand athletes. J Sports Med Phys fitness, 45(2), 183-187.

12. Doherty, T. J. (2003). Aging and sarcopenia. J Appl Physiol, 95, 1717-1727. doi:10.1152/japplphysiol.00347.2003

13. Ettinger, M. P. (2003). Aging bone and osteoporosis: strategies for preventing fractures in the elderly. Arch Intern Med, 163(18), 2237-2246.

14. Feland, J. B., \& Marin, H. N. (2004). Effect of submaximal contraction intensity in contract-relax proprioceptive neuromuscular facilitation stretching. Br J Sports Med, 38(4), E18.

15. Feland, J. B., Myrer, J. W., \& Merrill, R. M. (2001). Acute changes in hamstring flexibility: PNF versus static stretch in senior athletes. Phys Ther Sport, 2, 186-193. doi:10.1054/ptsp.2001.0076

16. Feland, J. B., Myrer, J. W., Schulthies, S. S., Fellingham, G. W., \& Measom, G. W. (2001). The effect of duration of stretching of the hamstring muscle group for increasing range of motion in people aged 65 years or older. Phys Ther, 81(5), 1110-1117.

17. Ferber, R., Osterning, L., \& Gravelle, D. (2002). Effect of PNF stretch techniques on knee flexor muscle EMG activity in older adults. J Electromyogr Kinesiol, 12(5), 391-397.

18. Franco, B. L., Signorelli, G. R., Trajano, G. S., \& Oliveira, C. G. (2008). Acute effects of different stretching exercises on muscular endurance. J Strength Cond Res, 22(6), 1832-1837.

19. Frontera, W. R., Hugues, V. A., Lutz, K. J., \& Evans, W. J. (1991). A crosssectional study of muscle strength and mass in 45 - to 78 -yr-old men and women. J Appl Physiol, 71(2), 644-650.

20. Gajdosik, R. L., Vander Linden, D. W., McNair, P. J., Riggin, T. J., Albertson, J. S., Mattick, D. J. et al. (2004). Slow passive stretch and release characteristics of the calf muscles of older women with limited dorsiflexion range of motion. Clin Biomech, 19(4), 398-406.

21. Gajdosik, R. L., Vander Linden, D. W., McNair, P. J., Riggin, T. J., Albertson, J. S., Mattick, D. J. et al. (2005). Viscoelastic properties of short calf muscle-tendon units of older women: Effects of slow and 
fast passive dorsiflexion stretches in vivo. Eur J Appl Physiol, 95(2-3), 131-139.

22. Gajdosik, R. L., Vander Linden, D. W., McNair, P. J., Williams, A. K., \& Riggin, T. J. (2005). Effects of an eight-week stretching program on the passive-elastic properties and function of the calf muscles of older women. Clin Biomech, 20(9), 973-983.

23. Gallon, D., Rodacki, A. L. F., Hernandez, S. G., Drabovski, B., Outi, T., Bittencourt, L. R. et al. (2011). The effects of stretching on the flexibility, muscle performance and functionality of institutionalized older women. Braz J Med Biol Res, 44(3), 229-235. doi:10.1590/So10o879X2011007500012

24. Godges, J. J., Mattson-Bell, M., Thorpe, D., \& Shah, D. (2003). The immediate effects of soft tissue mobilization with proprioceptive neuromuscular facilitation on glenohumeral external rotation and overhead reach. J Orthop Sports Phys Ther, 33(12), 713-718.

25. Goldspink, G. (1999). Molecular Mechanisms Involved in Determination of Muscle Fibre Mass and Phenotype. Adv Exerc Sports Physiol, 5 , 27-39.

26. Gomes, A. R., Cornachione, A., Salvini, T. F., \& Mattiello-Sverzut, A. C. (2007). Morphological effects of two protocols of passive stretch over the immobilized rat soleus muscle. J Anat, 210(3), 328-335.

27. Goodpaster, B. H., Chomentowski, P., Ward, B. K., Rossi, A., Glynn, N. W., Delmonico, M. J. et al. (2008). Effects of physical activity on strength and skeletal muscle fat infiltration in older adults: a randomized controlled trial. J Appl Physiol, 105, 1498-1503. doi:10.1152/ japplphysiol.90425.2008

28. Higgs, F., \& Winter, S. L. (2009). The effect of a four-week proprioceptive neuromuscular facilitation stretching program on isokinetic torque production. J Strength Cond Res, 23(5), 1442-1447.

29. Kabat, H. (1947). Studies of neuromuscular dysfunction, X: treatment of chronic multiple sclerosis with neostigmine and intensive muscle re-education. Perm Found Med Bull, 5(1), 1-14.

30. Kerrigan, D. C., Xenopoulos-Oddsson, A., Sullivan, M. J., Lelas, J. J., \& Riley, P. O. (2003). Effect of a hip flexor-stretching program on gait in the elderly. Arch Phys Med Rehabil, 84(1), 1-6.

31. Khamwong, P., Pirunsan, U., \& Paungmali, A. (2011). A prophylactic effect of proprioceptive neuromuscular facilitation (PNF) stretching on symptoms of muscle damage induced by eccentric exercise of the wrist extensors. J Bodyw Mov Ther, 15(4), 507-516.

32. Klein, D. A., Stone, W. J., Phillips, W. T., Gangi, J., \& Hartman, S. (2002). PNF Training and Physical Function in Assisted-Living Older Adults. J Againg Phys Activ, 10, 476-488.

33. Kofotolis, N., \& Kellis, E. (2006). Neuromuscular Facilitation Programs on Muscle Endurance, flexibility, and Functional Performance in Women With Chronic Low Back Pain. Phys Ther, 86(7), 1001-1012.

34. Lord, S. R., Sherrington, C., Menz, H. B., \& Close, J. C. (2000). Falls in older people: risk factors and strategies for prevention. Cambridge: Cambridge University Press.

35. Magnusson, S. P., Simonsen, E. B., Aagaard, P., Dyhre-Poulsen, P., McHugh, M. P., \& Kjaer, M. (1996). Mechanical and physiological responses to stretching with and without preisometric contraction in humans. Arch Phys Med Rehabil, 77(4), 373-378.

36. Mahomed, S., Al-Obaidi, S., \& Al-Zoabi, B. (2008). Outcome measures and psychomotor skills related to shoulder conditions for clinical orthopedic training. Med Princ Pract, 17(6), 481-485.

37. Manoel, M. E., Harris-Love, M. O., Danoff, J., V, \& Miller, T. A. (2008). Acute effects of static, dynamic, and proprioceptive neuromuscular facilitation stretching on muscle power in women. J Strength Cond Res, 22(5), 1528-1534.

38. Marek, S. M., Cramer, J. T., Fincher, A. L., Massey, L. L., Dangelmaier, S. M., Purkayastha, S. et al. (2005). Acute Effects of Static and Proprioceptive Neuromuscular Facilitation Stretching on Muscle Strength and Power Output. J Athl Train, 40(2), 94-103.

39. Mirek, E., Chwala, W., Longawa, K., Rudzinska, M., Adamkiewicz, P., \& Szczudilik, A. (2003). Proprioceptive neuromuscular facilitation method of therapeutic rehabilitation in the treatment of patients with Parkinson disease. Neurol Neurochir Pol, 37(Suppl 5), 89-102.

40. Nocon, M., Hiemann, T., Muller-Riemenschneider, F., Thalau, F., Roll, S., \& Willich, S. N. (2008). Association of physical activity with all-cause and cardiovascular mortality: a systematic review and meta-analysis. Eur.J.Cardiovasc.Prev.Rehabil., 15(3), 239-246. doi:10.1097/ HJR.obo13e3282f55eo9 [doi];00149831-200806000-00001 [pii]. Retrieved from PM: 18525377

41. Organización de Naciones Unidas. (2009). World Population Ageing.

42. Orsini, M., De Freitas, M. R. G., Oliveira, A. S. B., Silva, J. G., Leite, M. A. A., \& Nascimento, O. J. M. (2010). Efectos de un programa de facilitación neuromuscular propioceptiva sobre la amiotrofia focal benigna. Rev Neurol, 51, 317-318.

43. Profamilia. (2010). Encuesta Nacional de Demografía y Salud - ENDS.

44. Ramírez, J. F. (2011). Fuerza, funcionalidad y envejecimiento: consideraciones y perspectivas para su intervención (Primera ed.). Bogotá: Universidad Santo Tomás.

45. Ramírez, J. F., Da Silva, M., \& Lancho, J. (2007). Influencia de un programa de entrenamiento con saltos en la fuerza explosiva, la velocidad de movimiento y el equilibrio dinámico de varones longevos. Rev Esp Geriatr Geront, 42(4), 218-226.

46. Rebelatto, J. R., Castro, A. P., \& Paiva, A. (2007). Effect of the adult revitalization program on the occurrence of falls among its participants. Rev Bras Fisioter, 2, 383-389.

47. Rees, S. S., Murphy, A. J., Watsford, M. L., McLachlan, K. A., \& Coutts, A. J. (2007). Effects of proprioceptive neuromuscular facilitation stretching on stiffness and force-producing characteristics of the ankle in active women. J Strength Cond Res, 21(2), 572-577.

48. Ringsberg, K., Gerdhem, P., Johansson, J., \& Obrant, K. J. (1999). Is there a relationship between balance, gait performance and muscular strength in 75-year-old women? Age Ageing, 28(3), 289-293.

49. Rowlands, A., V, Marginson, V. F., \& Lee, J. (2003). Effect of isometric contraction duration during proprioceptive neuromuscular facilitation stretching techniques. Res Q Exerc Sport, 74(1), 47-51.

50. Rubenstein, L. Z. (2006). Falls in older people: epidemiology, risk factors and strategies for prevention. Age Ageing, 35(Suppl 2), ii37-ii41.

51. Ryann, E. E., Rossi, M. D., \& Lopez, R. (2010). The effects of the contract-relax-antagonist-contract form of proprioceptive neuromuscular facilitation stretching on postural stability. J Strength Cond Res, 24(7), 1888-1894.

52. Sharman, M. J., Cresswell, A. G., \& Riek, S. (2006). Proprioceptive neuromuscular facilitation stretching: Mechanisms and clinical implications. Sports Med, 36(11), 929-939.

53. Sherrington, C. S. (1908). On plastic tonus and proprioceptive reflexes. QJ Exp Physiol, 2, 109-156.

54. Shrier, I., \& Gossal, K. (2000). Myths and truths of stretching: Individualised recommendations for healthy muscles. Phys Sportsmed, 28(8), $57-63$. 
55. Sipila, S., \& Suominen, H. (1995). Effects of strength and endurance training on thigh and leg muscle mass and composition in elderly women. J Appl Physiol, 78, 334-340.

56. Tinetti, M. E., \& Kumar, C. (2010). The patient who falls: "It's always a trade-off". JAMA, 303(3), 258-266. doi:303/3/258 [pii];10.1001/ jama.2009.2024 [doi]. Retrieved from PM:20085954

57. Vandervoort, A. A. (2002). Aging of the human neuromuscular system. Muscle Nerve, 25(1), 17-25.

58. Voss, D., Lonta, M., \& Meyer, B. (1985). Proprioceptive Neuromuscular Facilitation: Patterns and Techniques. ( $3^{\circ}$ ed.). New York, NY: Harper \& Row.

59. Wallmann, H. W., Gillis, C. B., \& Martinez, N. J. (2008). The effects of different stretching techniques of the quadriceps muscles on agility performance in female collegiate soccer athletes: a pilot study. N Am J Sports Phys Ther, 3(1), 41-47.

60. Watt, R., Jackson, K., Franz, J. R., Dicharry, J., Evans, J., \& Kerrigan, D. C. (2011). Effect of a supervised hip flexor stretching program on gait in frail elderly patients. PM R, 3(4), 330-335.
61. Weening-Dijksterhuis, E., de Greef, M. H., Scherder, E. J., Slaets, J. P., \& van der Schans, C. P. (2011). Frail institutionalized older persons: A comprehensive review on physical exercise, physical fitness, activities of daily living, and quality-of-life. Am J Phys Med Rehabil, 90(2), 156168.

62. Weng, M. C., Lee, C. L., Chen, C. H., Hsu, J. J., Lee, W. D., Huang, M. H. et al. (2009). Effects of different stretching techniques on the outcomes of isokinetic exercise in patients with knee osteoarthritis. Kaohsiung J Med Sci, 25(6), 306-315.

63. Wolny, T., Saulicz, E., Gnat, R., \& Kokosz, M. (2010). Butler's neuromobilizations combined with proprioceptive neuromuscular facilitation are effective in reducing of upper limb sensory in late-stage stroke subjects: a three-group randomized trial. Clin Rehabil, 24(9), 810-821.

64. of static and PNF stretching exercises on range of motion and jump performance. J Bodyw Mov Ther, 13(1), 11-21.

65. Zhong, S., Chen, C. N., \& Thompson, L., V. (2007). Sarcopenia of ageing: Functional, structural and biochemical alterations. Rev Bras Fisioter, 11, 91-97.

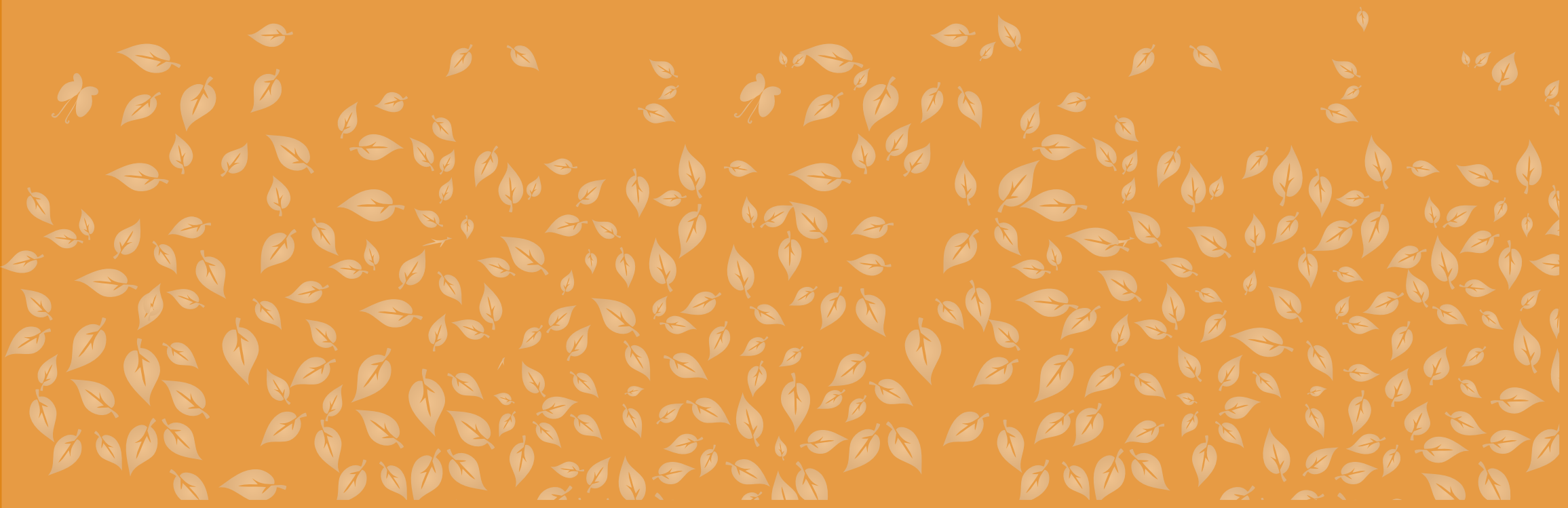

REV. COL. REH || Bogotá, Colombia || Volumen 11 || Página 6 - 13|| Noviembre 2012 || ISSN 1692 - 1879 